\title{
Is the Onset of Psychoactive Drug Effects Compatible with a Protein-Synthesis Mechanism?
}

\author{
MJ Kuhar*,' and AR Joyce' \\ 'Yerkes Regional Primate Research Center of Emory University, Division of Neuroscience, Atlanta, GA, USA
}

While many have suggested that protein synthesis may mediate the action of antipsychotic drugs, it is difficult to test. In this math modeling study it is found that the time course of action of the drugs are compatible with a protein-synthesis model and, furthermore, that the half-lives required by the model are indeed found in relevant proteins in the brain.

Neuropsychopharmacology (2003) 28, S94-S97. doi:I0.1038/s..npp. 1300140

Keywords: drug addiction; antipsychotic; protein synthesis; mathematical modeling; protein turnover; protein half-lives

\section{INTRODUCTION}

It is well known that many biological phenomena require protein synthesis for their manifestation. These include obvious processes such as growth, but also processes within the brain, such as memory. An intriguing problem in neuropsychiatry is the mechanism of action of antidepressants, antipsychotics, and addicting drugs. They are well known to be slow in onset, requiring from days to weeks to produce their full effect. While the mechanisms underlying this slow onset are unknown, elucidation of these mechanisms would be a significant advance, perhaps providing new targets for therapeutic development.

The hypothesis that protein synthesis could account for the action of antipsychotic and addicting drugs has been suggested in the past (Kuhar and Joyce, 2001). Recently, the rate of onset of drug effects was analyzed (Kuhar and Joyce, 2001) using a well-known mathematical model of protein synthesis described below. It was shown that the rates of onset of these drugs were compatible with changes in levels of proteins and that these proteins could theoretically mediate the clinical effect. This protein regulation hypothesis not only showed compatibility between the model of protein synthesis and the rate of onset of the drugs, but also showed that the half-lives of existing and known proteins were compatible with this model as well.

In this communication, the protein regulation hypothesis and its relation to the rate of onset of antipsychotic drugs are reviewed. We apply the simplest model first and then

*Correspondence: MJ Kuhar, Yerkes Regional Primate Research Center of Emory University, Division of Neuroscience, 954 Gatewood Rd NE, Atlanta, GA 30329, USA, Tel: + I 404727 1737, Fax: + | 404727 1737, E-mail: mkuhar@rmy.emory.edu

Received 01 September 2002; revised 03 November 2002; accepted 03 December 2002 examine increasingly complex interpretations that are compatible with the model and the data. Finally, we summarize the value of the model and how it would be used as perhaps one of many tools in studying the mechanism of action of these drugs. Nasar (1998) in her brilliant book has said 'The justification of all mathematical models is that, oversimplified, unrealistic, and even false as they may be in some respects, they force analysts to confront possibilities that would not have occurred to them otherwise.'

\section{MODEL OF PROTEIN SYNTHESIS}

It has been known for many decades that, in general, protein synthesis follows a zero-order process, whereas protein degradation is a first-order process (Berlin and Schimke, 1965; Hargrove, 1993; Schimke, 1973). This leads us to

$$
P_{t} / P_{\max }=\left(1-\mathrm{e}^{-0.693 t / h}\right),
$$

where $P_{t}$ is the protein level at time $t, P_{\max }$ is the maximal level of the protein and is equal to the synthesis rate $(r)$ divided by the degradation rate constant $(k)$, and where $h$ is the half-life of the protein under consideration. The curve derived from this equation as a function of $h$ is shown, for example, as the solid line in Figure 2a. Inspection of this equation indicates that the half-life, $h$, controls the relative rate of change of the level of the protein (Figure 2a). It takes about four half-lives for a protein to reach $94 \%$ of its final level and five half-lives to reach $97 \%$.

When several proteins are sequentially and interdependently involved, in other words where the level of a given protein depends on the synthesis of the previous protein, and where there are $n$ proteins in the series, the relevant 
equation is

$$
\begin{gathered}
P_{t n} / P_{\max n}=\left(1-\mathrm{e}^{-0.693 t / h 1}\right)\left(1-\mathrm{e}^{-0.693 t / h 2}\right) \cdots \\
\left(1-\mathrm{e}^{-0.693 t / h n}\right) .
\end{gathered}
$$

Equation 2 (2) should be regarded as the major analytic tool since it reverts to Equation 1 (1) when $n=1$, and because it embodies several reasonable alternatives that must be considered. The derivation of these equations, the shapes of the curves, their dependence on the various halflives, and their applicability to the hypothesis regarding the rate of onset of antipsychotic and addictive drugs has been explored in more detail previously (Kuhar and Joyce, 2001).

\section{HYPOTHESIS}

The hypothesis considered here is that clinical improvement (or simply change) is because of a protein(s), referred to as an 'effector(s).' The changing levels of the effector protein directly produces the proportionate corresponding clinical effect. The notion is that administration of a drug changes the gene expression, which in turn changes the protein levels (for a review of gene changes with addicting drugs, see Kuhar et al, 2001) which causes the clinical change.

\section{EXAMPLE OF DATA ANALYSIS}

Figure 1a shows the rate of clinical improvement of a group of schizophrenics treated with clozapine (Lieberman et al, 1994). Figure 1b, where the data are shown again, includes smooth curves that were generated by computer using Equation 1 (1) with various half-lives of 20, 30, and 40 days. The smooth curves show the rate of change of theoretical protein levels. The curve derived using a 30-day half-life appears to fit the data points best, but curves from other half-lives would fit within reasonable errors as well. The implication according to the hypothesis is that an effector protein characterized by these half-lives could mediate the clinical improvement.

Thus, the rate of change of protein levels according to the model seems compatible with the rate of improvement in these patients. Also, different proteins with half-lives that vary considerably may still be within the inherent variability of the data. An important practical point is that brain proteins with these half-lives would have to exist to further test the hypothesis and model, and they do (see below).

A word about errors in measuring $P_{t} / P_{\max }$ and their impact on $h$ :

For example, what is the effect on $h$ when $P_{t} / P_{\max }$ might vary by $\pm 20 \%$ ? We can approach this by considering a variation of Equation 1 (1):

$$
h_{2} / h_{1}=\ln \left(1-P_{t 1} / P_{\max 1}\right) / \ln \left(1-P_{t 2} / P_{\max 2}\right) \text {. }
$$

When $P_{t 1} / P_{\max 1}=1 / 2$, and $P_{t 2} / P_{\max 2}$ varies by $\pm 20 \%$, the ratios of the half-lives, $h_{2} / h_{1}$ are 0.76 and 1.36 from the above equation. Therefore, if the $h$ of a measured curve is calculated to be 10, where error in $P_{t} / P_{\max }$ range from + to $-20 \%$, then the half-life could range from 7.6 to 13.6 days, a ratio of almost two-fold. Thus, errors in measurement of
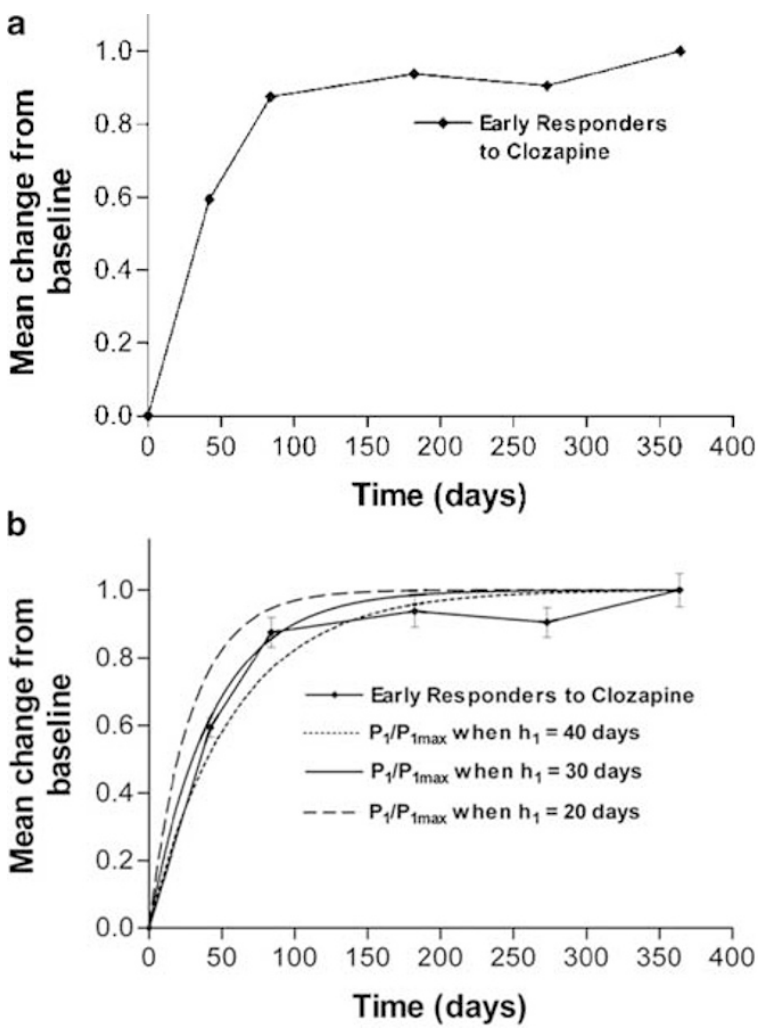

Figure I Time course of clinical improvement of a group of schizophrenics as described by Lieberman et al (Lieberman et al, 1994). A. Reproduction of original data. B. Superposition of curves using Equation I (I), suggesting that the half-life of the 'effector' protein is about 30 days. Curves of different half-lives were used to develop an appreciation of how variability in the data affects the half-lives. See text for details.

$P_{t} / P_{\max }$ can have a significant impact on the range of halflives that must be considered.

\section{INTERPRETATION}

It is clear that the time course data can be fit to an exponential curve compatible with the involvement of a single protein with a given half-life (Figure 1). However, further inspection and examination of the model reveals that additional interpretations are possible and must be considered to explore fully the biologic and mechanistic possibilities. The first additional possible interpretation is simply that the curve generated by changing levels of a single effector protein with a given half-life can actually be produced by the additive effects of more than one protein of about the same half-life. As shown in Figure 2a, a curve for a single protein is indistinguishable from a curve where two proteins with about the same half-life are all effectors and produce effects that are additive. Therefore, even though the simplest exponential can fit the curve, the involvement of more than one protein whose effects are additive, that is multiple effectors, must be considered as a real possibility.

Yet additional interpretations are possible when we consider Equation 2 (2), which describes the production of a final effector protein that is dependent on the production of previous proteins that in turn regulate the 


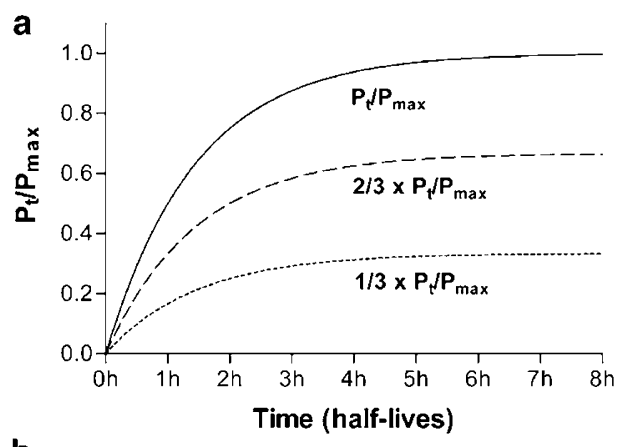

b

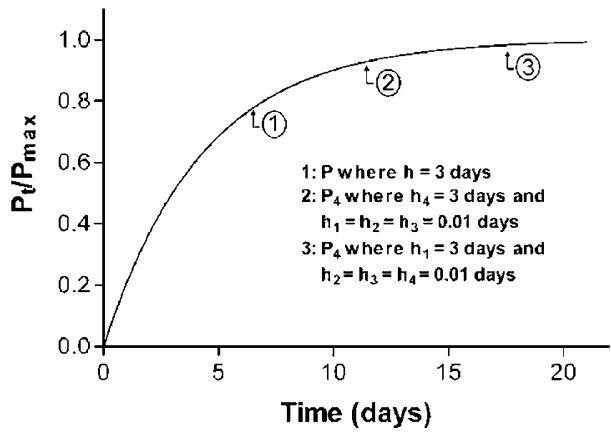

c

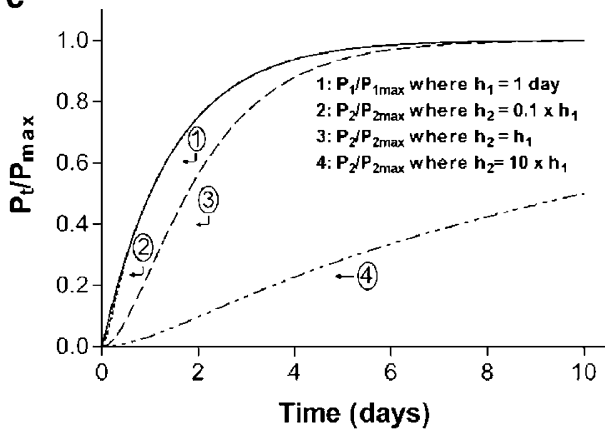

levels of the next. A concrete example of this might be a series of proteins where the first is a transcription factor that produces and regulates the formation of subsequent effector proteins. Figure $2 \mathrm{~b}$ shows three different curves that appear as one because they overlap and are indistinguishable. Curve 1 is a simple exponential derived from Equation 1 (1), where the half-life of the theoretical protein is 3 days. However, Curves 2 and 3 derive from Equation 2 (2), where there are four different, dependent, and sequentially expressed proteins, the last one being the effector. Curve 2 shows the case where the effector protein half-life is 3 days and the half-lives of the other precursor proteins are much smaller, 0.01 days. In this case, the very short half-life proteins are 'invisible' in the process and only the longest half-life protein has an influence on the time course of the process. In Curve 3, the first protein has a half-life of 3 days and the subsequent three proteins, including the effector, have very small half-lives, but the curve is dominated by the first, longest half-life protein. It is clear, considering Curves 2 and 3, that the longest half-life protein dominates the shape of the curve, no matter where it occurs in the sequence. This is both a shortcoming of the method, as well as a strength, since the long half-life proteins, that is the slowest to reach the maximal effect, are often of most interest. When half-lives of different and sequentially regulated proteins are more similar than different, there is a lag in the rise of the protein levels (Figure 2c). Indeed, a lag in the rise of levels can easily be interpreted and modeled by Equation 2 (2) (Kuhar and Joyce, 2001).

The above analysis indicates that clinical improvement in these schizophrenics could involve a protein with a half-life of about 30 days. Does any such protein(s) exist in the human brain? The answer is yes. While very few half-lives of proteins have been measured in humans, MAO-B has a halflife of about 40 days (Fowler et al, 1994) in the human brain. If we consider that 'synaptic remodeling' is needed for the clinical effect, then formation of synaptic proteins could be rate limiting. Botulinum toxins target synaptic proteins involved in neurotransmitter release, and the time of recovery of function after administering these toxins to humans is of the order of a month or more, suggesting that their half-lives in vivo are also of the same order of

Figure 2 Theoretical curves using Equations I and 2, (I) and (2), that help to explain the mechanistic alternatives that might underlie curves shown in Figure I (following text and figures from Kuhar and Joyce, 200 I). The time courses of protein concentrations $\left(P_{t} / P_{\max }\right)$ are shown after a change in the synthesis or degradation rate of the protein. (a) The concentration of a single protein (solid line) is determined by the model presented in Equation I ( 1 ) of the text (a single protein as the effector), which shows that 50,75, 87, and $94 \%$ (designated on the ordinate in fractions rather than percent) of the maximum concentrations are reached by one, two, three, and four half-lives (h), respectively. If a decrease in protein concentration was occurring, the curve would be drawn downwards. If the $y$-axis reflected the clinical effects as a result of a protein increase, the shape of the 'effect' curve would be equivalent to the protein concentration curve. The effect of a single protein could also be produced by adding the effects of two other proteins that have the same half-lives and the concentrations of which start rising at the same time, just as the dashed lines add to produce the solid line. These proteins could be different proteins that have different mechanisms but the same effect, or similar proteins (isoforms) that elicit the same effect. (b) Data obtained when four proteins are sequentially regulated and the shape of the final effect curve is strongly influenced by the longest half-life protein, even if this protein is not the effector. The curve shown is actually three different curves that overlap $(I-3)$. Curve $\mid$ is for a single protein (Equation I (I)) where the half-life is 3 days. When the half-lives of the first three proteins are very small compared with that of the effector (Curve 2), the influence of the first three proteins on the shape of the curve is insignificant. Similarly, when the half-life of the first precursor, $\mathrm{PI}$, is much longer than that of P2, $\mathrm{P} 3$, and P4 (Curve 3), PI dominates the time course and the influence of the other three proteins is not apparent. (c) Curves where the effector protein is the last in a series of sequentially regulated proteins and where the half-lives of the different proteins are more similar than for the data in part (b). Two proteins, $\mathrm{PI}$ and $\mathrm{P} 2$, are considered where the ratio of the half-lives vary; $\mathrm{hl}$ is I day in all cases and $\mathrm{PI}$ controls the concentrations of P2 as described in the main text. Curve I (Equation I (I)) is for the single protein PI only. Curves 2-4 (from Equation 2 (2)) show P2 concentrations when $\mathrm{hl} / \mathrm{h} 2$ varies. When the half-life of $\mathrm{Pl}$ is much larger than $\mathrm{h} 2$, the overall curve is largely determined by $\mathrm{PI}$ (Curve 2 is not significantly different from Curve I), and there is almost no delay in the early time course for P2 (Curve 2). However, as the half-life of PI becomes relatively short compared with that for $\mathrm{P} 2$, a lag in the rise of the curve becomes evident (Curves 3 and 4). This lag in the curve for P2 reflects the time required for $\mathrm{PI}$ to rise to significant concentrations before it can produce significant concentrations of P2 (see text for details). Curves were generated using a desktop PC computer with Microsoft Excel, S-Plus 2000, and Graph Pad Prism 3.0. Abbreviations: P, protein concentration; $P_{t}$, the concentration of protein and time $t ; P_{\max }$, the maximum concentration of the protein. 
magnitude (Brin, 1997; Edgar, 2001; Eleopra et al, 1998). Thus, it appears that relevant human brain proteins have half-lives of the magnitude required for the data to fit the model. Since half-lives may be species dependent (Kuhar and Joyce, 2001), additional half-life measurements will be needed in humans in order to apply the model to humans.

Since the basic assumption of the model used here employs a zero-order synthesis rate and a first-order degradation rate, it is fortuitous that other identically regulated processes can be added to the model (Hargrove, 1993). Drug levels in the brain are determined by a similar process (Goldstein et al, 1974), as are the mRNA levels within cells (Hargrove, 1993). Thus, while the model is described above as only dealing with changes in the levels of proteins, we could easily include changing drug levels and mRNA levels as factors in Equation 2 (2), and the half-lives derived from the analysis may apply to other processes as well. However, since the overall curve will reflect the longest half-life steps in the series, these faster steps may be 'invisible' and negligible when the overall process is measured. As Hargrove (Hargrove, 1993), for example, has pointed out in a very general analysis, the half-time of transcription and of mRNA decay in cytoplasms is of the order of 3 and 10-1000 min, respectively; these are indeed very short compared to the half-time of the recovery process in Figure 1. If the half-time of the antipsychotic drugs in the brain were 4 days, it still would have a minor influence on the overall curve as shown by Equation 2 (2), where an overall half-time of 30 days is implied.

The crux of this approach is to fit curves generated by the model to the experimental data. If there is a reasonable fit, one can then consider all of the theoretical possibilities that permit the fit and then plan additional experiments or considerations based on those possibilities. It is clear that a fit does not prove the validity of the model or a specific aspect of the model, but rather allows these to be considered. In this sense, it is a tool to be used in studying the possible effectors of processes in brain. While this modeling approach is ambitious, includes many significant simplifications and assumptions, and uses data without a highly detailed time course, it nevertheless provides a reasonable fit to the available data and requires a half-life that is within the realm of reality. Many additional studies will be needed to assess fully the utility of the model, and perhaps to expand the model.

\section{ACKNOWLEDGEMENTS}

We acknowledge the support of NIH Grants RR00165, DA00418, and DA10732.

\section{REFERENCES}

Berlin CM, Schimke RT (1965). Influence of turnover rates on the responses of enzymes to cortisone. Mol Pharmacol 1: 149-156.

Brin MF (1997). Botulinum toxin: chemistry, pharmacology, toxicity, and immunology. Muscle Nerve 6(Suppl): S146-S148.

Edgar TS (2001). Clinical utility of botulinum toxin in the treatment of cerebral palsy: comprehensive review. J Child Neurol 16: 37-46.

Eleopra R, Tugnoli V, Rossetto O, De Grandis D, Montecucco C (1998). Different time courses of recovery after poisoning with botulinum neurotoxin serotypes A and E in humans. Neurosci Lett 256: 135-138.

Fowler JS, Volkow ND, Logan J, Wang GJ, MacGregor RR, Schyler D (1994). Slow recovery of human brain MAO B after L-deprenyl (Selegeline) withdrawal. Synapse 18: 86-93.

Goldstein A, Aronow L, Kalman SM (1974). Principles of Drug Action: The Basis of Pharmacology. John Wiley \& Sons: New York.

Hargrove JL (1993). Microcomputer-assisted kinetic modeling of mammalian gene expression. Faseb J 7: 1163-1170.

Kuhar MJ, Joyce AR (2001). Slow onset of CNS drugs: can changes in protein concentration account for the delay? Trends Pharmacol Sci 22: 450-456.

Kuhar MJ, Joyce A, Dominguez G (2001). Genes in drug abuse. Drug Alcohol Depend 62: 157-162.

Lieberman JA, Safferman AZ, Pollack S, Szymanski S, Johns C, Howard A (1994). Clinical effects of clozapine in chronic schizophrenia: response to treatment and predictors of outcome. Am J Psychiatry 151: 1744-1752.

Nasar S (1998). A Beautiful Mind. Touchstone: New York.

Schimke RT (1973). Control of enzyme levels in mammalian tissues. Adv Enzymol Relat Areas Mol Biol 37: 135-187. 